\title{
Nachdenken über Unendlichkeit
}

\author{
Ein Vorschlag für einen fächerübergreifenden Unterricht in \\ Mathematik und Religion mit philosophischen Aspekten
}

Jürgen Maaß*

https://doi.org/10.53349/resource.2021.i16.a926

\section{Zusammenfassung}

Mit diesem Text möchte ich dazu motivieren, sich in der Schule und außerhalb mit dem Thema „Unendlichkeit“ zu beschäftigen. Dazu wähle ich Zugänge über Mathematik, Philosophie und Religion, die je nach Interesse und Möglichkeiten genutzt werden können. Diese Thematik hat in der Geschichte viele Menschen beschäftigt; sehr viele bedeutende Werke dazu finden sich in den Bibliotheken. Dennoch soll die Ehrfurcht davor oder die Tatsache, dass immer Fragen offenbleiben werden, niemanden davon abhalten, sich überhaupt mit der Thematik zu beschäftigen. Im Gegenteil: Vieles lässt sich schon mit jüngeren Schüler*innen so diskutieren, dass Wissenszuwachs und Neugier auf mehr erreicht werden können. In den Text eingewoben sind konkrete Unterrichtsvorschläge.

$\begin{array}{ll}\text { Schlüsselwörter: } & \text { Keywords: } \\ \text { Unendlich } & \text { Infinity } \\ \text { Interdisziplinärer Zugang } & \text { Interdisciplinary approach } \\ \text { Unterrichtsvorschläge } & \text { Teaching proposals }\end{array}$

\section{Einleitung}

„Der Weltraum. Unendliche Weiten. Wir schreiben das Jahr 2200...“ Wer kennt diese Sätze aus dem Intro der „Star Trek“ Filme nicht? Viele Millionen Menschen verfolgen gespannt am Bildschirm die Bemühungen der Crew des Raumschiffes „Enterprise“, die „unendlichen“ Weiten des Weltraumes zu erkunden. Sehr viel weniger Menschen beteiligen sich mit großem eigenen Interesse daran, im Mathematikunterricht selbst die Vielfalt von faszinierenden Phänomenen rund um Unendlichkeit zu erkunden. Häufig wird auch im Unterricht kaum eine Möglichkeit dazu geboten, weil das Unendliche nicht zum Thema gemacht, sondern auf ein paar Regeln zum Umgang mit Grenzwerten reduziert wird. Ziel dieses Beitrages ist der mathematikdidaktische Versuch, durch die Beschäftigung mit verschiedenen Aspekten der Unendlichkeit Lernenden einen für sie neuen Blick auf Mathematik zu ermöglichen. Damit ist die Hoffnung verbunden, dass zumindest einige der Lernenden motiviert werden, sich nachhaltig und selbstständig in dieser Thematik und damit in Mathematik weiterzubilden.

Ausgangspunkt für den Start ins Unendliche kann die „einfache“ Frage sein, ob das Weltall „tatsächlich“ unendlich groß ist. Die Suche nach einer Antwort führt in die Physik, Astronomie oder Kosmologie und vermutlich ist sie in der Schulklasse umstritten. Am Anfang gab es - so die derzeit populäre Theorie - einen Urknall, dann eine Phase der Expansion und irgendwann fällt alles wieder in sich zusammen - vielleicht, um mit dem nächsten Urknall ein neues Universum zu beginnen. In der Physik herrscht diese Theorie nicht mehr vor. Im Jahre 2011 wurde der Nobelpreis für Physik an drei Forscher vergeben: „Sie haben mehrere Dutzend explodierende Sterne, Supernovae genannt, untersucht und entdeckt, dass sich das Universum mit einer immer höher werdenden Geschwindigkeit ausdehnt. Die Entdeckung kam sogar für die Preisträger selbst völlig überraschend“ (https://www.nobelprize.org/prizes/physics/2011/press-release/). Muss das Universum deshalb unendlich groß werden? Vielleicht - wenn es sich über eine unendlich lange Zeit immer schneller ausdehnt. Wer dieses Thema vertieft behandeln möchte, gerät wegen der hier als Argumente verwendeten mathematischen Modelle schnell

\footnotetext{
* Johannes Kepler Universität Linz, Altenbergerstraße 69, 4040 Linz.

E-Mail: familie.maasz@aon.at
} 
über das Schulniveau hinaus. Eine gut verständliche Zusammenfassung findet sich bei Wikipedia (https://de.wikipedia.org/wiki/Kosmologie\#Expansion des Universums), eine mit Physik fachwissenschaftlich fundierte etwa bei Matthias Bartelmann (2019).

Gibt es mehrere solcher Universen wie das unsere? Eventuell sogar unendlich viele? Die Science-Fiction-Fans in der Schulklasse werden eingeladen, darüber zu berichten, was sie gelesen oder gesehen haben. Vermutlich gibt es keine endgültige Entscheidung, aber eine Zeit intensiver Diskussion, in der auch Argumente fallen, die logisch mit vermuteten Eigenschaften von Unendlichkeit verknüpft sind. Diese Argumente sollten gesammelt und dokumentiert werden.

Auf jeden Fall bietet eine Diskussion über die Weiten des Weltalls die Möglichkeit, sehr große und unendlich große Zahlen besser zu unterscheiden. Wie groß ist das Weltall? Wenn es nicht unendlich groß ist, hat es eine endliche Größe. Im letzten Jahrtausend wurde sie (etwa von David Deutsch 1996) als Kugel mit einem Radius von mehr als 45 Milliarden Lichtjahren angesehen. Die sichtbare Masse beträgt ca. $10^{53} \mathrm{~kg}$. Damit können wir ein wenig rechnen: Wenn ein Lichtstrahl von einem Ende zum anderen Ende 90 Milliarden Jahre (oder 100 Milliarden Jahre oder gar - bei einem großen Fehler in der Theorie - 10000 Milliarden Jahre) braucht, wie viele Kilometer oder Nanometer hat das Licht dann zurückgelegt? Zur Erinnerung: Ein Lichtjahr entspricht 9,46 Billionen Kilometer $\left(9,46 \cdot 10^{12} \mathrm{~km}\right)$. Welches Volumen hat die Riesenkugel? Wie viel Platz hat ein Kilogramm sichtbare Materie durchschnittlich in dieser Kugel? Wie viele Atome enthält ein Kilogramm Materie? Wie viele Atome enthält die Riesenkugel? All diese und viel mehr ähnliche Rechnungen können wir mit einem schlichten Taschenrechner und mit gerundeten Zahlen sogar mit einem Stift und Papier ohne große Probleme beantworten. Selbst wenn wir uns um den Faktor 10 oder sogar um den Faktor eine Milliarde verrechnen, ist es im Hinblick auf das eingangs erwähnte Ziel nicht schlimm. Alle Rechnungen führen zu endlich großen Zahlen. Wir können uns $10^{20} \mathrm{~km}$ ebenso schwer vorstellen wie $10^{21} \mathrm{~km}$ oder $10^{30} \mathrm{~km}$. Wir wissen aber von diesen drei und allen anderen ähnlichen Entfernungen, dass sie endlich und nicht unendlich groß sind. Vielleicht beflügelt es die Phantasie der Lernenden, wenn sie sich bei der Erläuterung der Eigenschaften einer Geraden nicht nur vorstellen, dass sie ein wenig über den Rand der Tafel oder des Bildschirms hinausreichen, sondern beliebig viel weiter als der am weitesten entfernte sichtbare Stern am Himmel.

\section{Erstes Beispiel: Parallele Geraden schneiden sich im Unendlichen?}

Wie verhalten sich in unendlicher Entfernung zwei parallele Geraden, die noch viel weiter von uns weg reichen als jedes sichtbare Objekt am Nachthimmel ist? Sind sie noch parallel, wenn sie den Orion oder die Andromeda Galaxis passieren? Lichtstrahlen werden durch Schwerkraft beeinflusst (sind also keine Geraden). ${ }^{1}$ Also ersinnen wir eine Situation, in der sie sich aus formalen Gründen irgendwo ganz weit entfernt treffen müssen. Wir konstruieren ein Dreieck mit zwei rechten Winkeln. Für den dritten Winkel sind dann noch null Grad übrig, zwei Seiten dieses Dreiecks sind also parallel. Sie sind Strahlen und liegen auf parallelen Geraden, die sich nicht treffen dürfen, aber treffen müssen, damit das Dreieck seine dritte Ecke bekommt, einen Eckpunkt. Was nun?

Wenn wir ein solches Dreieck auf Papier zeichnen, erhalten wir eine Strecke zwischen zwei Eckpunkten und zwei parallele Strahlen, die von den Eckpunkten ausgehen und sich auf dem Papier nicht treffen. Das Bild ändert sich nicht, wenn wir ein sehr großes Blatt Papier, die Tafel oder den Flur vor dem Klassenraum zum Zeichnen verwenden. Auf dem Heimweg fahren wir vielleicht mit der Straßenbahn oder der Eisenbahn und überlegen uns, dass die Schienen bis auf Kurven immer parallel sind und sich nie treffen. Sonst entgleist der Zug. Selbst auf einer sehr langen Strecke wie New York - San Francisco oder Moskau - Wladiwostok kommt ein Zug gut an, weil die Schienen immer parallel zueinander verlaufen (in der Realität gibt es Kurven und wie bei allen technischen Konstruktionen kleine Toleranzen im Abstand, die wir als Passagiere vermutlich nicht bemerken) und ihren Abstand nicht verändern. Mit dem Beispiel im Kopf können wir in der nächsten Stunde herausarbeiten, dass der entscheidende Punkt offenbar der unendliche Abstand des möglichen Schnittpunktes ist. Endlich große Darstellungen von einem solchen Dreieck bleiben offen, ohne einen Schnittpunkt der beiden parallelen Geraden, auch wenn „endlich“ sehr groß ist (wie das Beispiel Eisenbahnschienen zeigen soll). Im Unendlichen, in einer unendlich weit entfernten Stelle passiert vielleicht etwas, das wir nicht sehen können.

Nun können wir mit Plato ${ }^{2}$ annehmen, dass es geometrische Objekte wie Punkte, Strecken oder Dreiecke nicht in der Realität gibt, sondern nur als Ideen, als Objekte, die wir gedanklich erfassen und mehr oder weniger schlecht in der Realität darstellen können. Sobald wir einen Punkt zeichnen, malen wir einen Klecks, einen kleinen Kreis oder ein Kreuz, von dem wir sagen, im Schnittpunkt der beiden Linien liegt der Punkt, den wir meinen. Aber sobald wir einen sichtbaren Punkt zeichnen, ist es kein Punkt, sondern eine Fläche. Ein Punkt ist nach Definition nicht sichtbar, er ist unteilbar und unendlich klein, ohne Ausdehnung. Plato würde also vermutlich sagen, dass 
unser rätselhaftes Dreieck durch irgendwelche Veranschaulichungen wie Linien auf Papier oder Schienen ohnehin nicht richtig dargestellt wird. Nur unser Geist kann die Idee eines solchen Dreiecks korrekt erfassen. Das aber verschärft unser Problem mit dem Unendlichen: Unser Geist hat Schwierigkeiten, die sich wiedersprechenden Ideen bzw. Definitionen von einem Dreieck mit Winkelsumme $180 \mathrm{Grad}$ und parallelen Geraden, die sich nicht schneiden, gleichzeitig richtig zu erfassen. Die Schwierigkeiten lassen sich bekanntlich lösen, wenn wir unser Dreieck auf einer Kugel betrachten oder gleich den Sprung zu nicht-euklidischen Geometrien wagen. Letzteres geht aber nicht an der Schule.

Ich beschreite jetzt nicht den üblichen universitären mathematischen Weg, durch geeignete Ergänzungen zu den Basisaxiomen einer Geometrie (vgl. W. Blaschke 2013) solche Probleme auszuschließen, sondern schlage vor, es im Unterricht mit jüngeren Schüler*innen bei der Feststellung zu belassen, dass wir im Umgang mit „Unendlich“ auf Fragen stoßen, die wir nicht einfach beantworten können. Zwar gibt es Erklärungs- und Antwortmöglichkeiten, aber häufig auch einen - mehr oder weniger großen - Rest von Unglauben oder Unsicherheit. Dieser Rest kann dazu führen, die Finger und den Geist von solchen Fragen, also dem Unendlichen, fernzuhalten oder sich intensiver damit zu beschäftigen.

\section{Zweites Beispiel: Fußballfans und andere gleichmächtige Mengen}

Ein zweites, ähnliches Beispiel für etwas ältere Schüler*innen soll ebenfalls verdeutlichen, dass im Unendlichen Phänomene auftreten können, die wir mit dem endlichen Hausverstand nicht nachvollziehen können. Nehmen wir an, in der Stadt findet ein Fußballspiel statt, ein Derby zweier Mannschaften aus dieser Stadt. Auf dem Weg zum Stadion sehen wir immer wieder Gruppen von Fans, die mit ihren Vereinsfarben geschmückt sind. Eine Gruppe trägt grün, die andere violett. Wir haben den Eindruck, dass etwa gleich viele Menschen grüne oder violette Farben tragen. Im Stadion wird der Eindruck bestätigt: Damit es nicht zu Auseinandersetzungen zwischen den Fangruppen kommt, ist je eine Hälfte des Stadions für die Anhänger*innen eines Clubs reserviert. Beide Hälften sind ausverkauft - es sind ziemlich genau gleich viele Fans beider Vereine im Stadion.

Nun machen wir einen kleinen Abstraktionsschritt: Wir nehmen an, die Fans des grünen Vereins werden gezählt, indem jedem Fan eine gerade Zahl zugeordnet wird: $2,4,6,8,10, \ldots n * 2$. Die Fans mit den violetten Vereinsfarben erhalten die ungeraden Zahlen: $1,3,5,7, \ldots\left(m^{*} 2-1\right)$. Nun ist ganz offensichtlich, dass für den Fall $n=m$ beide Vereine gleich viele Fans (im Stadion) haben. Jetzt aber kommt der Schock im Klassenraum: Wir behaupten, dass - wenn wir $\mathrm{m}=\mathrm{n}$ unendlich groß werden lassen - genauso viele Fans von jedem der beiden Vereine wie Menschen insgesamt im - fiktiven unendlichen großen - Stadion sind. Im realen Stadion sind es offensichtlich doppelt so viele Menschen wie Fans je eines Vereins. Was Merkwürdiges und kaum Glaubhaftes mag passieren, wenn wir in Gedanken unendlich viele Menschen ins Stadion lassen, von denen je die Hälfte Fan eines der beiden Vereine ist?

Können wir als Lehrperson die Schüler*innen überzeugen, indem wir mit Bezug auf Cantor (vgl. Oliver Dieser 2010) die Gleichmächtigkeit von Mengen durch die Existenz einer bijektiven Abbildung begründen? Wir ordnen jeder natürlichen Zahl $n$ die natürliche Zahl $2 * n$ zu und erhalten eine bijektiven Abbildung. Es gibt demnach genauso viele gerade (oder ungerade) Zahlen wie natürliche Zahlen insgesamt.

Stellen wir uns vor (oder arrangieren es im Klassenraum): Die Fans beider Vereine stellen sich in zwei Reihen nebeneinander auf. Je zwei Personen, die nebeneinander stehen, reichen sich die Hand. Für den Fall, dass es in der Schulklasse eine ungerade Anzahl von Schüler*innen gibt, kann eine Person als neutraler Beobachter (bzw. als neutrale Beobachterin) daneben stehen. Die beiden Reihen sind gleich lang, haben gleich viele Personen (Elemente), von denen je zwei durch ihre Hände sichtbar verbunden sind. Das Händereichen veranschaulicht auch schön die bijektive Abbildung zwischen den beiden Reihen. Ganz offensichtlich sind in beiden Reihen gleich viele Personen. ABER: Wenn beide Reihen unendlich lang sind, sollen in einer Reihe ebenso viele Personen stehen wie in beiden zusammen?

Wie beim oben thematisierten Dreieck mit zwei rechten Winkeln passiert das Unerwartete dadurch, dass $n$ unendlich groß werden darf. Für endliche Anzahlen (auch bei großen Zahlen wie den Fans im Stadion) ist anschaulich alles klar. Bei einer unendlich großen Anzahl, der Menge aller natürlichen Zahlen, geht eben diese anschauliche Klarheit verloren. Ein unerklärliches (oder besser: nur durch eine abstrakte Definition von Gleichmächtigkeit begründetes) Phänomen tritt auf. 


\section{Vollständige Induktion kann ebenfalls verwirren}

Wer Mathematik studiert hat, erinnert sich: Alle Mengen, die gleichmächtig zu jener der natürlichen Zahlen sind, heißen abzählbar (unendlich groß). Das führt zur Frage nach noch größeren Mengen, überabzählbar genannt und gleichmächtig zu jener der reellen Zahlen oder einer noch größeren Menge (etwa der Potenzmenge der reellen Zahlen). Bevor ich darauf eingehe, erwähne ich noch ein Beispiel, an dem die besondere Eigenschaft der natürlichen Zahlen, ihre Abzählbarkeit, für eine besondere Art von Beweis genutzt wird: Die vollständige Induktion. Wenn eine Eigenschaft für eine natürliche Zahl bewiesen wird und gezeigt wird, dass diese Eigenschaft für eine Zahl $n+1$ gilt, wenn sie für $n$ gilt, dann gilt sie für alle natürlichen Zahlen.

Als Beispiel für einen Beweis nutze ich eine Anekdote, die über den berühmten Mathematiker Carl Friedrich Gauß (1777-1855) berichtet wird. Er soll als Siebenjähriger die Aufgabe „Addiere alle Zahlen von 1 bis 100 “ sehr schnell gelöst haben. Er hat gesehen, dass sich je zwei Zahlen $(1+100,2+99,3+98, \ldots)$ zu gleich großen Paaren (mit dem Wert 101) zusammenfassen lassen. Es gibt 50 solcher Paare, also ist das Resultat $101 * 50=5050$. Ich mache nun aus dieser genialen Lösungsidee eine Formel, die ich dann mit vollständiger Induktion beweise.

Behauptung: die Summe aller natürlicher Zahlen von 1 bis $n$ ist $(1+n)$ mal $n / 2$. Für das Beispiel $n=100$ stimmt die Formel offenbar, da $101 * 50=5050$ wie oben berechnet. Nun kommt der eigentliche Trick beim Beweis durch vollständige Induktion. Ich möchte zeigen, dass die Formel für $n+1$ gilt und darf dabei voraussetzen, dass sie für $\mathrm{n}$ richtig ist. Ich starte also mit der Summe aller natürlicher Zahlen von 1 bis $\mathrm{n}+1$ und hoffe zeigen zu können, dass sie für sie gilt: Sie ist gleich $(1+n+1) *(n+1) / 2$.

Die Summe aller natürlicher Zahlen von 1 bis $n+1=(1+n) * n / 2+(n+1)$. Dabei habe ich die Summe der Zahlen bis $n$ mit Hilfe der Induktionsvoraussetzung, dass die Formel für alle natürlichen Zahlen bis $n$ richtig ist, durch die entsprechende Formel ersetzt. Nun fehlt noch ein wenig elementare Algebra, um zu zeigen, dass $(1+n) * n / 2+$ $(n+1)=(1+n+1) *(n+1) / 2$. Ich schreibe nur einen Zwischenschritt auf, der durch Ausmultiplizieren beider Terme entsteht: $n^{2} / 2+3 / 2 * n+1$.

Nach einigen Übungen und Beispielen haben die Schüler*innen die Beweismethode „Vollständige Induktion“ verinnerlicht: Zuerst wird die Behauptung für eine bestimmte natürliche Zahl, sei es 1 oder 564373854, bewiesen. Dann zeigen wir, dass die Behauptung für jede größere natürliche Zahl richtig ist, indem wir von $n$ auf $n+1$ schließen. Nun beweise ich auf diese Weise, dass unendlich viele Taschentücher in einen Reisekoffer passen. Zunächst öffne ich den Koffer und lege ein Taschentuch hinein. Damit habe ich die Induktionsbasis. Dann stelle ich mir vor, dass es mir gelungen ist, eine bestimmte Zahl $n$ Taschentücher in den Koffer zu legen. Nun öffne ich den Koffer noch einmal und füge ein einzelnes Taschentuch hinzu. Hat jemand Zweifel daran, dass es mir gelingen wird, noch ein Taschentuch in den Koffer zu packen? Sicher nicht. Wer schon einmal einen Koffer gepackt hat, weiß, dass für ein zusätzliches Taschentuch immer noch ein Platz über ist - im Zweifelsfall muss ich ein ganz klein wenig drücken.

Und nun? Ich habe mit vollständiger Induktion bewiesen, dass ich unendlich viele Taschentücher in einen gewöhnlichen (also nicht unendlich großen) Reisekoffer packen kann. Nebenbei habe ich auch bewiesen, dass es unendlich viele Taschentücher gibt. Das widerspricht der Anschauung, also begeben wir uns auf Fehlersuche.

Hat Ihre Schulklasse es herausgefunden? Wenn ich mit ein wenig Druck ein zusätzliches Taschentuch in den Koffer packe, verändere ich die Ausgangsbedingungen. Nehmen wir an, ich drücke die im Koffer vorhandenen zehntausend Taschentücher so zusammen, dass das Zehntausenderste noch hineinpasst. Dann habe ich auch die Ausgangsbedingungen meiner Induktionsannahme verändert: Die Taschentücher, die nun im Koffer sind, haben im Durchschnitt 1/10000 weniger Volumen - es sind also andere Taschentücher. Damit habe ich den Gang meines Beweises verlassen. Ich kann nun über die etwas kleineren Taschentücher eine Aussage machen und versuchen sie zu beweisen - oder ich freue mich darüber, den Fehler im Beweisgang gefunden zu haben und belasse es dabei.

\section{5 Überabzählbar unendlich: In unendlich vielen Stufen ins Religiöse?}

G. Cantor hat im Zuge seiner Überlegungen zur Mächtigkeit von Mengen nicht nur gezeigt, dass es genauso viele rationale Zahlen (Brüche) wie natürliche Zahlen gibt, sondern auch, dass die reellen Zahlen um eine ganze Stufe mehr sind, eben überabzählbar. Ein nachvollziehbarer Weg von natürlichen zu reellen Zahlen ist die Potenzmenge, also die Menge aller Teilmengen der natürlichen Zahlen. Eine einzige reelle Zahl erhalte ich z. B., wenn ich alle natürlichen Zahlen als Ziffern hintereinander schreibe ${ }^{3}$ : 1,12345678910111213141516.... Selbstverständlich kann ich auch eine andere Reihenfolge wählen, nur die geraden Zahlen oder die Vielfachen 
einer beliebigen anderen natürlichen Zahl verwenden. Ich kann mich auch nach einem Rechenergebnis richten, etwa dem Ergebnis einer Division wie 17 geteilt durch 3, die zu einer periodischen Dezimalzahl führt: 5,6666...

Die reellen Zahlen $\mathrm{R}$ haben viele faszinierende Eigenschaften, die ich hier nicht behandeln möchte. Im Hinblick auf die Frage „unendlich/gleichmächtig“ erwähne ich nur ein erstaunliches Phänomen: Im Intervall von Null bis Eins (oder jedem anderen endlich großen Intervall) liegen ebenso viele Elemente wie in der Menge der reellen Zahlen selbst. Es gibt also unendlich viele Teilmengen von $\mathrm{R}$, die jeweils gleichmächtig zu $\mathrm{R}$ sind. Das passt wieder gar nicht zu unseren Erfahrungen mit Personengruppen. Wenn wir eine Gruppe mit 24 Personen (also etwa unsere Schulklasse) in Teilgruppen aufteilen (also etwa 8 Arbeitsgruppe mit je 3 Schüler*innen), bleibt die Gesamtzahl der Personen gleich. Wenn wir umgekehrt mehrere Gruppen (etwa alle 16 Schulklassen unserer Schule) mit jeweils 24 Schüler*innen auf dem Schulhof versammeln, haben wir insgesamt 16 mal 24 Schüler*innen (und nicht nur 24!) auf dem Schulhof versammelt. Das ist offenbar wie bei den Fußballfans: Wenn es ins Unendliche geht, passt unsere Erfahrung mit endlich großen Zahlen nicht zur Definition von „gleichmächtig“. Hier mag das Phänomen sogar noch weniger fassbar sein, weil wir es bei R und Intervallen als Teilmengen von R mit überabzählbar großen Mengen zu tun haben. Und überabzählbar unendlich ist noch weniger erfahrbar als abzählbar unendlich.

Ein in der Schule gut verwendbares Beispiel für eine Funktion, die ein Intervall auf ganz R bijektiv abbildet, ist die Tangensfunktion. Wenn ein*e Schüler*in nicht glaubt, dass sich ein Intervall, auf dem eine Tangensfunktion definiert ist, mit einem Faktor so dehnen oder strecken kann, dass auch ein sehr kleines oder sehr großes Intervall genau einmal ausgefüllt wird, bietet sich eine interaktive Übung mit GeoGebra an.

Cantor hat die Idee, Potenzmengen von Mengen auch im Hinblick auf Ihre Mächtigkeit zu untersuchen und zu ordnen, bis zum Ende in der Unendlichkeit verfolgt (vgl. Georg Cantor: 1872-1884). Die abzählbar unendlichen Mengen wie die der natürlichen Zahlen haben die Mächtigkeit Aleph null. Die nächstgrößere Stufe, überabzählbare Mengen wie die reellen Zahlen, Aleph eins. Wer die Mächtigkeit steigern will, kann immer wieder - unendlich oft! - die Potenzmenge der vorhandenen Menge bilden, und landet bei ... Gott?

Bevor ich den Schritt in die Theologie wage, noch ein Hinweis zum Mathematikunterricht. Selbstverständlich ist dies nicht der erste Vorschlag, Unendlichkeit im Mathematikunterricht zu behandeln. Ich verweise exemplarisch auf das Heft 112 der Zeitschrift „Mathematik lehren“ (hgv. Beutelsbacher/Weigand 2002) zum Thema „unendlich“.

\section{Unendlich aus theologischer Sicht}

Wir verdanken G. Cantor, dass er eine mathematisch nachvollziehbare Ordnung in die Stufen der Unendlichkeit gebracht hat - der Zusammenhang über Potenzmengen ist nachvollziehbar, auch wenn viele Phänomene im Zusammenhang mit „unendlich“ Erstaunen auslösen. Lange Zeit vor Cantor - und mit einem weniger ausdifferenzierten Begriff von „unendlich“ - haben aber schon viele Menschen über „unendlich“ nachgedacht. Ein typischer Gedankengang ist uns vermutlich gut vertraut: Wenn es ein Wesen gibt, das unendlich mächtig, allwissend und ewig seiend ist, dann muss es notwendig auch existieren. Solch ein Beweisgang ist als „ontologischer Gottesweis" in die Philosophiegeschichte eingegangen".

Nun schlage ich vor, nicht einfach zu berichten, dass etwa I. Kant solche Beweise für die Existenz Gottes als tautologische Zirkelschlüsse kritisierte (man steckt als Voraussetzung eines Beweises eine Aussage hinein, die man hinterher aus den Voraussetzungen herleitet). Für ein besseres Verständnis des Unendlichen scheint es mir möglich, gerade aus den Charakterisierungen eines Wesens, das Unendlichkeit verkörpert, etwas über „unendlich“ zu lernen. Also suchen wir solche Beschreibungen und thematisieren wir sie im fächerübergreifenden Unterricht Mathematik/Religion.

Im Koran finden sich 99 Gottbeschreibungen, die mit ALL beginnen. Einige davon zitiere ich hier ${ }^{5}$

\begin{tabular}{|c|c|c|}
\hline 15 & القهار & al-Qahhār \\
\hline 19 & العليم & al-'Alīm \\
\hline 57 & المحصى & al-Muḥșī \\
\hline 63 & القيوم & al-Qayyūm \\
\hline 64 & الواجد & al-Wāğid \\
\hline 70 & المقتدر & al-Muqtac \\
\hline 73 & الأول & al-Awwal \\
\hline 74 & الأخر & al-Ahir \\
\hline 96 & الباقي & al-Bāqī \\
\hline
\end{tabular}

der Alles-Bezwinger

der Allwissende

der alles Aufzeichnende

der allein Stehende, der Ewige

der alles Bekommende und Findende

der alles Bestimmende

der Erste ohne Beginn

der Letzte ohne Ende

der ewig Bleibende" 
In der Bibel (besser in jeder Übersetzung der Bibel - siehe https://www.bibleserver.com/search) finden sich ähnlich Beschreibungen ${ }^{6}$ Gottes, insbesondere auch für die Allmacht. „Sir 19,20: Alle Weisheit besteht in der Furcht des Herrn, und zu aller Weisheit gehört das Tun des Gesetzes [und die Erkenntnis seiner Allmacht]. “ Der Bibelserver liefert auch gleich die Textstelle mit. Andere Texte finden sich insbesondere in den Psalmen:

„Der HERR ist mächtig, groß ist sein Ruhm, unermesslich ist seine Macht!“ (Psalm 145,3)

„Unser Herr ist gewaltig, groß ist seine Macht, seine Einsicht hat keine Grenzen." (Psalm 147,5)

„Gepriesen sei der Herr, der Gott Israels, vom Anfang bis ans Ende der Zeiten." (Psalm 106,48)

\section{Damit ergibt sich genug Material für den Unterricht. Aber wie soll es eingesetzt werden?}

Mir scheint es gut, eine Beschreibung auszuwählen und damit genauer zu arbeiten. Als Beispiel nehme ich „allwissend“. Was weiß ein allwissendes Wesen? Offenbar nicht nur die Aufgaben in der nächsten Schularbeit, alle Namen und Telefonnummern aus dem Telefonbuch oder alle Internetadressen. Wer alles weiß, kennt nicht nur alle Nachbar*innen, Freund*innen und Verwandte der Schüler*innen in der Schulklasse, sondern alle Menschen, die jetzt leben, irgendwann gelebt haben und - hier mag diskutiert werden - alle Menschen, die noch nicht geboren wurden. Von allen Menschen weiß dieses Wesen, was sie getan, gedacht, gewollt, richtig oder falsch gemacht haben. Mit anderen Worten: dieses Wesen hat nicht nur sehr viele Informationen, es ist auch fähig, weise und gerecht zu urteilen. Alles über alle Lebewesen auf diesem Planeten (oder allen Planeten aller Sterne) zu wissen, bedeutet ein sehr großes, aber noch kein unendliches Wissen. Wird das Wissen unendlich groß, wenn ich alles Wissen über die belebte und unbelebte Natur hinzunehme? Über die Vergangenheit und Zukunft? Wenn ich die Lebensdauer unseres Universums mit vielen Milliarden Jahren annehme und mir überlege, dass ein allwissendes Wesen über jedes Atom darin in jeder noch so kleinen Zeiteinheit während der gesamten Lebensdauer des Universums alles weiß, bleibe ich dennoch bei einer endlich großen Menge an Informationen. Allwissend ist mehr!

Nehmen wir an, wir finden einen Weg, dem allwissenden Wesen unendlich viel Wissen zuzuordnen, dann stoßen wir auf die nächste Frage: Ist ein Wesen, das unendlich viel weiß, allwissend? Nein, hier helfen uns Cantors Unterscheidungen. Wenn ein Wesen, das unendlich viel weiß, dieses Wissen ordentlich nummeriert und dabei auf eine abzählbare Menge von Informationen kommt, weiß es offenbar unendlich viel nicht - all jenes Wissen fehlt, das in seiner Ordnung auf den Plätzen steht, die im Zahlensystem von irrationalen Zahlen eingenommen wird. Allwissend ist also noch mehr als unendlich viel wissend.

Nun prüfen wir den Begriff „Allwissenheit“ ein wenig aus einer Richtung, die für die Theologie immer eine besondere Herausforderung war, nämlich der Logik. Didaktisch bietet sich hier eine Kleingruppenarbeit an: Überlegt Euch bitte, was logisch alles aus der Eigenschaft „Allwissenheit“ folgt? Gibt es logische Grenzen oder Widersprüche?

Viele Punkte können erarbeitet und besprochen werden. Relativ einfach erscheint die Möglichkeit, ein allwissendes Wesen kennt ganz genau die Vergangenheit eines jeden Menschen. Das passt gut zu der Vorstellung, dass dieses Wissen beim Jüngsten Gericht verwendet wird, um ein gerechtes Urteil über jeden Menschen und seine (Un-)Taten zu fällen. Mehr Probleme macht die Überlegung, dieses Wesen weiß auch alles über die Zukunft. In der Prädestinationslehre von Calvin und Zwingli findet sich dafür eine in der Geschichte der christlichen Religionen recht pointierte Ausprägung. „Sowohl für Zwingli als auch für Calvin ist klar, dass der Mensch nicht wirklich über einen freien Willen verfügt. Der Mensch kann sein Glück nicht selber wählen, sondern es ist Gott, der ihm das Heil schenkt. Gemäß Zwingli erwählt die Barmherzigkeit Gottes Menschen, worauf der gerechte Gott sie von ihrer Schuld freispricht und als seine Kinder adoptiert. Von den Verworfenen ist bei ihm nur am Rande die Rede. Zwingli lehrt nicht, dass Gott auch Menschen zur Verdammnis erwähle, während Calvin meint, dass Gottes Gerechtigkeit Menschen zur Verwerfung erwähle (doppelte Prädestination). ${ }^{77}$

Im 16. Jahrhundert fanden die reformierten Kirchen, die auf Calvin und Zwinglis Lehren beruhten, einen großen Zulauf. Die anderen christlichen Kirchen lehnen die Lehre von der doppelten Prädestination aus verschiedenen Gründen ab. Im fächerübergreifenden Unterricht bietet es sich an, die vielen Argumente für und gegen diese Lehre z.B. in Form von Referaten oder einer gut vorbereiteten Podiumsdiskussion (einzelne Schüler*innen vertreten je eine vorher festgelegte Position) zu reflektieren. Ich möchte an dieser Stelle nicht weiter darauf eingehen, aber noch eine zusätzliche Position einbringen. 
Die Logik verlangt dann nämlich, dass dieses allwissende Wesen schon zum Anbeginn der Zeit und der Welt ganz genau wusste, was alles passieren wird. Gibt es demnach genau eine Zukunft, die diesem Wesen schon bekannt ist, uns aber nicht? Haben wir keinen Einfluss auf die Zukunft, egal was wir tun oder nicht tun? Glauben wir nur, dass wir irgendwelche freien Entscheidungen fällen? Oder fällen wir selbst frei unsere Entscheidungen ohne aber daran zu denken, dass dieses Wesen schon seit Milliarden von Jahren weiß, was wir im nächsten Augenblick tun oder nicht werden? Verweilen wir einen Augenblick bei diesem Gedanken. Für das, was wir im nächsten Augenblick tun wollen, haben wir meist eine begrenzte Anzahl von Möglichkeiten: Weiter an diesem Text arbeiten, atmen, auf Geräusche achten, zum Kühlschrank gehen, das Fenster öffnen, ... Für das, was wir nicht tun wollen oder können, gibt es viel mehr Möglichkeiten: Nicht zum Mond fliegen, zur Venus oder zum Jupiter, nicht das Sonnensystem verlassen, um viele andere zu erkunden. Wenn wir die Orte, die wir alle nicht besuchen, mit Hilfe von reellen Zahlen mit Koordinaten versehen, erhalten wir sofort unendliche viele Orte. Wenn wir die Überlegung auf alle Lebewesen zu jedem Zeitpunkt in Vergangenheit, Gegenwart und Zukunft ausdehnen, erhalten wir überabzählbar unendlich viele Informationen. Gibt es bei Allwissenheit einen Relevanzfilter? Sortiert das Wesen alles aus, was nicht relevant ist, um weniger Daten zu speichern und auszuwerten? Oder spielt das bei Allwissenheit keine Rolle?

\section{Exkurs zur Unterrichtsmethode}

Hier wie an anderen Stellen im hier von mir vorgeschlagenen Unterricht gibt es im Unterschied zur typischen Rechenaufgabe im Mathematikunterricht keine eindeutige Lösung, die mit "richtig" oder "falsch" beurteilt werden kann. Wir sind nicht allwissend und haben keine Information darüber, wie ein allwissendes Wesen mit dem Wissen umgeht. Deshalb können wir nicht einfach "richtig" oder "falsch" auf die Frage antworten, ob und wie ein solches Wesen über die Relevanz seines Wissens entscheidet und welche Konsequenzen solche Entscheidungen eventuell haben. Aber das Nachdenken und die Diskussion über solche Fragen erweitert ganz sicher den Horizont der Mitwirkenden.

\section{Die Monaden von Leibniz: Ein Modell für die Allwissenheit des Schöpfers?}

Auf der Suche nach einem Modell, das uns plausibel erklärt, wie ein allwissender Schöpfer dafür sorgen könnte, dass die vorhergesehene Zukunft auch tatsächlich wie nur ihm allein schon bekannt tatsächlich eintritt, bin ich auf G. Leibnitz (1646-1716) und seine Monaden gestoßen. Leibniz hat „Monaden“ in 90 Punkten definiert und beschrieben ${ }^{8}$. Was sind Monaden? Sie sind „die wahrhaften Atome der Natur" (Punkt 3) und haben "keine Ausdehnung, keine Gestalt und keine mögliche Teilbarkeit" (Punkt 3). Monaden können nur durch Erschaffung beginnen (Punkt 6). Sie sind fensterlos, d. h. kein Mittel kann „erklären, wie eine Monade in ihrem Inneren durch ein anderes erschaffenes Ding beeinflusst oder verändert werden kann“ (sie sind "fensterlos" - Punkt 7).

Fragen wir nach dem Sinn von Monaden und ihren Aktivitäten (z. B. als aus Monaden zusammengesetzten Körpern), so erfahren wir von Leibniz: „Daher muss der letzte Grund der Dinge in einer notwendigen Substanz liegen, in der als in der Quelle das Einzelne der Veränderungen nur wesentlich enthalten ist, und eben diese Substanz nennen wir Gott." (Punkt 38).

Vor Milliarden von Jahren hat - so Leibnitz - ein allwissendes und allmächtiges Wesen (von ihm Gott genannt) unendlich viele unendlich kleine Monaden geschaffen und jeder einzelnen Monade ein fixes Programm mitgegeben, das ganz exakt sagt, wo sich die Monade in all den Jahren zu jedem beliebigen Zeitpunkt aufhalten soll, ob sie sich mit anderen Monaden zusammen zu einem Objekt (z.B. einem Ball) zusammentun soll, was dann dieses Objekt tun soll (z.B. als Fußball beim Elfmeter ins Tor fliegen) und welche Funktion all die Monaden nachher übernehmen sollen. Besonders spannend wird die Diskussion, wenn zur Sprache kommt, dass auch alle Personen, die an der Diskussion teilnehmen, aus Monaden zusammengesetzt sind, denen Gott schon vor sehr langer Zeit ein fixes Programm mitgegeben hat, dass sie abarbeiten, ohne auf Einflüsse von außen zu achten (,fensterlos"). Was bedeutet das für diese Personen (und alle anderen) im Hinblick auf ihr Leben, ihre Zukunft, ihre Entscheidungen? Wer Leibnitz folgt, muss zu dem Schluss kommen, dass alles fix vorgegeben ist (von Gott unveränderbar determiniert). Das kann zu heftigen Diskussionen führen! Wenn ich am Nachmittag ein Spiel mit Würfeln spiele, wusste Gott das schon und hat festgelegt, dass ich mit dem fünften Wurf eine Sechs würfle, mit dem fünfundzwanzigsten eine drei usw. Ebenso hat er vorherbestimmt, dass ich überhaupt mit meinen Freunden dieses Würfelspiel zur Unterhaltung wähle (und kein Kartenspiel oder Computerspiel). Er hat auch den Regen am 
Nachmittag vorprogrammiert, der Grund dafür ist, dass wir im Zimmer bleiben und nicht draußen Fußball spielen. Selbstverständlich hat er dann auch vorbestimmt, wer meine Freunde oder Freudinnen sind, wo ich wohne, wer meine Eltern sind und ganz zum Schluss (für mich) wann und wie ich sterben werden.

Ein so vollständig vorbestimmtes Leben ist für viele Menschen eine unangenehme oder sogar unerträgliche Vorstellung. Daran ändert auch die Überlegung nichts, dass in einem solchen Fall kein Mensch für irgendetwas verantwortlich ist. Wenn ein jeder Mensch „nur" einer eine lange Zeit vorher festgelegten Lebenslinie folgt, ohne darauf Einfluss zu haben, wo und wie diese Linie verläuft, kann jeder Mensch nur als teilnehmender Beobachter über ethische Fragen nachdenken oder Verantwortung tragen.

Auch mit dieser entlastenden Zusatzüberlegung bleibt - so behaupte ich - Leibnitz mit seinen Monaden (ebenso wie andere deterministische Theorien) unpopulär. Die Willensfreiheit und ihr entscheidender Einfluss auf unseren Lebensweg ist den meisten Menschen sehr wichtig.

\section{Willensfreiheit und Unendlichkeit}

In der Menge Bibel ${ }^{10}$ findet sich (Sir 17,5) die Textstelle „Urteilskraft (oder Willensfreiheit?) und Sprache und Augen, Ohren und ein Herz zum Denken gab er ihnen. “Wenn Gott den Menschen die Fähigkeit und Möglichkeit gab, selbst zu erkennen und zu entscheiden, wirft das eine Vielzahl von Fragen auf, die (und deren Beantwortung) in der Theologie ganze Bibliotheken füllt. Der Bezug zu „meinem“ Thema Unendlichkeit ist ein sehr wenig wichtiger Randaspekt, der von folgender Frage ausgeht: Kann ein Mensch unendlich viele Wünsche haben? Solange wir davon ausgehen (müssen oder dürfen), dass Menschen sterblich sind, kommen wir zu dem Schluss, dass alles Menschenwerk endlich ist: Auch, wenn jemand sehr viel und weit reist, legt dieser Mensch in seinem Leben nur eine endliche Entfernung zurück. Das gilt sogar für Reisen in Science-Fiction Romanen. Selbst wer schneller als das Licht von einem Stern zum nächsten reist, legt insgesamt nur eine endliche Entfernung zurück. Eine ähnliche Überlegung gilt für einen Menschen, der viel liest oder auf andere Weise Informationen sammelt. Auch mithilfe eines computergestützten Zusatzgedächtnisses bleibt die Menge der Informationen endlich groß, die ein Mensch in seinem Leben sammeln und verarbeiten kann. Gilt dieses Argument auch für Wünsche? Wenn die Formulierung eines Wunsches (oder vieler Wünsche) eine endliche Zeit braucht (was aufgrund der bisherigen Gehirnforschung der Fall ist), kann in einer endlichen Lebensspanne nur eine endliche Anzahl von Wünschen formuliert werden. Unendlich viel kann nur gewünscht werden: Ein unendlich langes Leben, unendlich viel Gold, unendlich viel Wissen ... Aber auch der gütigste Gott oder die beste Fee kann einen solchen Wunsch nicht erfüllen, oder?

\section{Unendliche Macht - Allmacht}

Auch über die Allmacht Gottes ist viel mehr nachgedacht und geschrieben worden, als hier auch nur angedeutet werden kann. Nehmen wir nur den „biblischen Text über die Schöpfung der Welt (Gen 1,1-2,3)“: Ein allmächtiger Gott kann selbstverständlich vor etwa 7000 Jahren den Entschluss gefasst haben, die Welt, die wir kennen, samt allen Fossilien, Milliarden von Jahren alten Sternen und Gesteinen zu erschaffen. Wenn er das nicht könnte, wäre er offenbar nicht allmächtig, sondern „nur“ sehr mächtig. Wenn wir uns fragen, weshalb er das getan haben könnte, hören wir vielleicht aus den USA eine Antwort von einer bestimmten Gruppe von Menschen, die sagt: Unsertwegen! Wir wollen Recht haben! Wer sich ernsthafter mit der Schöpfung auseinandersetzen will, sollte unbedingt das Buch von Franz Gruber (Im Haus des Lebens. Eine Theologie der Schöpfung, Verlag Pustet, Regensburg 2001) lesen.

Ich möchte hier beim mathematikdidaktischen Zugang bleiben und vorschlagen, in einer Schulklasse im Zusammenhang mit Allmacht über Logik und Unendlichkeit nachzudenken. Typische Fragen dazu sind die nach dem Beginn und einem möglichen Ende. Wie beginnt die Geschichte? War das allmächtige Wesen schon immer da? Wenn es ewig ist, hat es keinen Beginn oder ist es ewig nach dem Beginn? Wenn es begonnen hat: Ist es seinerseits erschaffen worden? Oder irgendwie entstanden? Logisch am einfachsten wäre es wohl, sich seine Existenz auf einer Zeitachse von minus endlich bis plus unendlich vorzustellen. Ein Ende eines allmächtigen und ewigen Wesens wäre unlogisch.

Kann es zwei oder mehrere solcher Wesen geben? Offenbar nicht - sie würden sich wechselseitig in ihrer Allmacht einschränken. Das legt aber den Gedanken nahe, dass die Allmacht doch beschränkt ist: Das allmächtige Wesen kann kein anderes allmächtiges Wesen schaffen. Deshalb ist Gottes Sohn Jesus Christus nicht ein anderes 
Wesen, sondern Teil des einen allmächtigen Wesens (gemeinsam mit einem dritten Teil der Trinität, dem Heiligen Geist), oder?

\section{Schlussbemerkung}

Aus meiner Sicht hat eine Schulklasse viel erreicht, wenn sie nach dem hier vorgeschlagenen gemeinsamen und Fächer übergreifenden Nachdenken über Unendlichkeit neugierig geworden ist. Zumindest einige Schüler*innen sollten auch außerhalb der Schule weiter darüber nachdenken. In Bezug auf Mathematik ist Cantor und anderen das Verdienst zuzurechnen, das Unendliche für die Zwecke der Mathematik handhabbar zu machen. Mit bestimmten einschränkenden und genauer definierten Bedingungen ist das Unendliche so nützlich wie das Feuer. Es hilft uns etwa, Analysis mit all ihren reichhaltigen Ergebnissen zu betreiben.

P.S.: Ich danke Dr. W. Burgstaller für seine hilfreichen Hinweise zu theologischen Fragen und Dr. C. Spreitzer für seine hilfreichen Hinweise zu physikalischen Fragen.

\section{Literatur}

Matthias Bartelmann (2019): Das kosmologische Standardmodell. Grundlagen, Beobachtungen und Grenzen, Springer Heidelberg

Albrecht Beutelspacher, Hans-Georg Weigand (2002): Endlich...unendlich! Mathematik lehren, Heft 112 Friedrich in Velber Verlag in Zusammenarbeit mit Klett

W. Blaschke (2013): Projektive Geometrie, Springer-Verlag, ISBN 3-0348-6932-0

Georg Cantor (1984): Über unendliche, lineare Punktmannigfaltigkeiten. Arbeiten zur Mengenlehre aus dem Jahren 1872-1884 (= Teubner-Archiv zur Mathematik. Bd. 2, ISSN 0233-0962) ). Herausgegeben und kommentiert von G. Asser. Teubner, Leipzig

David Deutsch (1996): Die Physik der Welterkenntnis. Auf dem Wege zum universellen Verstehen. Birkhäuser, Berlin ISBN 3-7643-5385-6

Franz Gruber (2001): Im Haus des Lebens. Eine Theologie der Schöpfung, Verlag Pustet, Regensburg

Oliver Dieser (2010): Einführung in die Mengenlehre: Die Mengenlehre Georg Cantors und ihre Axiomatisierung durch Ernst Zermelo. 3. Auflage. Springer, Berlin/Heidelberg, ISBN 978-3-642-01444-4, doi:10.1007/9783-642-01445-1

Platon (2017): Der Staat (Reclams Universal-Bibliothek) Gebundene Ausgabe 2017

\footnotetext{
${ }^{1}$ Das Weltall ist kein euklidischer Raum. Das zeigt sich besonders deutlich in der Nähe von schwarzen Löchern.

${ }^{2}$ Im „Staat“ führt Sokrates aus, „dass sie (die Geometer, Anm. JM) sich der sichtbaren Gestalten bedienen und immer von diesen reden, während den eigentlichen Gegenstand ihres Denkens nicht diese bilden, sondern jene, deren bloße Abbilder diese sind. Denn das Quadrat an sich ist es und die Diagonale an sich, um derentwillen sie ihre Erörterungen anstellen, nicht aber dasjenige, welches sie durch Zeichnung entwerfen." (Platon 2017, Der Staat 511a).

${ }^{3}$ So eine Konstruktion wird auch als Champernowne-Zahl bezeichnet, siehe https://de.wikipedia.org/wiki/Champernowne-Zahl

${ }^{4}$ Der ontologische Gottesbeweis schlussfolgert aus dem bloßen Gedanken oder Begriff "Gott", d.h. a priori, auf die Existenz Gottes. Anselm von Canterbury entwarf die erste und bekannteste Version des ontologischen Gottesbeweises:

(P1) Gott ist definiert als das vollkommenste Wesen, über das hinaus nichts Vollkommeneres gedacht werden kann.

(P2) Existenz ist eine vollkommenheitsfördernde Eigenschaft. D.h.: Wenn man zwei Wesen $X$ und $Y$ hat, die sich in allen Eigenschaften gleichen, abgesehen davon, dass $X$ existiert und $Y$ nicht, dann ist $X$ vollkommener als $Y$.

(P3) Wenn Gott nicht existieren würde, könnte man sich vorstellen, dass er vollkommener wäre, als er ist (P2). Das steht im Widerspruch zu (P1).

(K) Also: Gott existiert.

https://www.philoclopedia.de/was-kann-ich-wissen/gottesbeweise/ontologischer-gottesbeweis/

${ }^{5}$ https://de.wikipedia.org/wiki/Gottes_sch\%C3\%B6ne_Namen
} 
${ }^{6}$ Wer etwa Bibelstellen zum Begriff „ewig“ sucht, erhält folgende Verweise:

Lutherbibel 2017

85 Stellen

Elberfelder Bibel

227 Stellen

Einheitsübersetzung 2016

192 Stellen

Zürcher Bibel

142 Stellen

Neue Genfer Übersetzung

109 Stellen

Gute Nachricht Bibel

57 Stellen

Neues Leben. Die Bibel

116 Stellen

Hoffnung für Alle

69 Stellen

Neue evangelistische Übersetzung

78 Stellen

Menge Bibel

98 Stellen

Schlachter 2000

124 Stellen

${ }^{7}$ https://www.zhref.ch/themen/reformationsjubilaeum/allgemeine-informationen/huldrych-zwingli/zwinglilexikon-von-a-bis-z-1/lexikon-c/calvin

${ }^{8}$ Siehe https://www.hermetik-international.com/mediathek/historische-schriften-der-mystik/gottfried-

wilhelm-leibniz-die-monadologie/. Zugegriffen: 14. Juli 2020.

${ }^{10}$ https://de.wikipedia.org/wiki/Hermann_Menge 